\title{
6 Ecology and Evolution of Plasticity
}

\author{
Emilie C. Snell-Rood and Sean M. Ehlman \\ University of Minnesota
}

\section{CONTENTS}

6.1 Introduction

6.2 Selection for Plasticity: Environmental Variation and

Environmental Extremes

6.2.1 Theory Emphasizes the Importance of Environmental Variation.... 141

6.2.2 Empirical Data Suggests Environmental Variation Is Important, but Not Entirely Predictive

6.2.3 Evolution of Plasticity as a Byproduct of Strong Directional Selection.

6.2.4 Organismal Traits Structure Environmental Variation 143

6.2.5 Selection on Plasticity Is Limited by Cue Reliability.... 144

6.3 Costs and Limits Select against Plasticity, but Are Often Lower than

Expected

6.3.1 Conflict between Theoretical Expectations of Costs and Empirical Observations

6.3.2 When We See Costs, Where Are They Coming From?

6.3.3 Why Don't We See Costs of Plasticity More Consistently Across Studies?

6.4 Future Directions in Ecology and Evolution of Plasticity

6.4.1 Plasticity and Cryptic Genetic Variation in Novel Environments .... 150

6.4.2 Costs of Plasticity and Genetic Assimilation in Novel Environments... 151

6.4.3 Feedbacks in the Evolution of Plasticity: When the Environment Evolves

6.4.4 Evaluating Null Models in the Evolution of Plasticity ..... 152

6.4.5 Developmental Realism in Evolutionary Models: Predicting Diversification and the Emergence of Specialists and Generalists

6.5 Conclusions

References 153

\subsection{INTRODUCTION}

Adaptive phenotypic plasticity allows organisms to cope with a range of environments through adjustments in a range of traits, from gene expression and physiology, to behavior and morphological development (West-Eberhard 2003; Sultan 2015; 
Gilbert and Epel 2015; see also Pfennig 2021; Sultan 2021 in this volume). The degree of adaptive plasticity varies both within and across species, and such variation in plasticity has implications for understanding why some organisms survive and diversify in novel environments (Sol et al. 2005; Pfennig et al. 2010; Diamond and Martin 2021; Levis and Pfennig 2021). Thus, biologists have long been interested in the ecological and evolutionary drivers of plasticity. Why are some organisms more phenotypically flexible, while others are relatively more fixed in trait expression? The parallel literature on variation in niche breadth has considered a similar question: Why do some organisms use a range of environments and resources while others are more specialized (Futuyma and Moreno 1988; Richards et al. 2006; Sexton et al. 2017)?

In this chapter, we focus on the ecological and evolutionary causes of variation in adaptive phenotypic plasticity versus a more specialized strategy (Figure 6.1). We first review the evolutionary drivers of plasticity, evaluating the classic role of environmental variation relative to other factors such as novel, extreme environments. Second, we review the costs and limits associated with phenotypic plasticity and review how

(a)

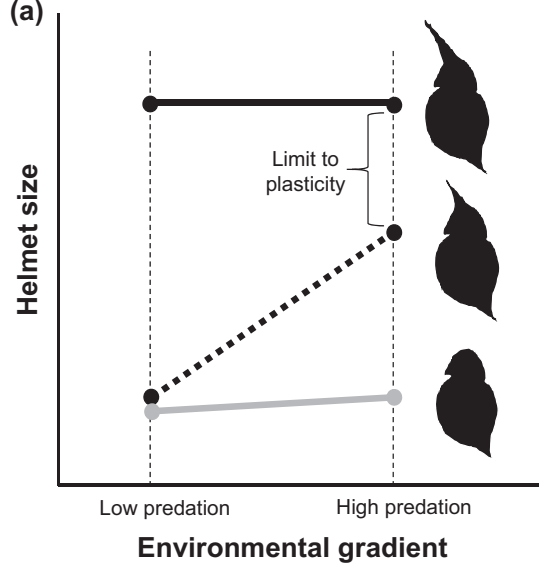

(b)

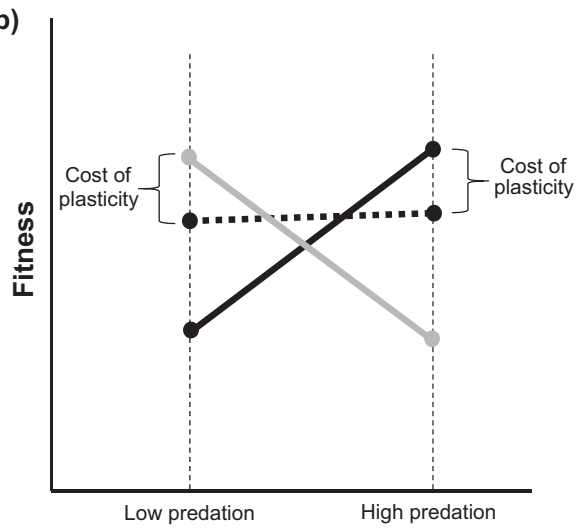

Environmental gradient

FIGURE 6.1 Variation in adaptive phenotypic plasticity. To illustrate the costs and benefits of plasticity, consider the zooplankton Daphnia, which induces a protective spine in response to predator cues. Panel (a) uses reaction norms to illustrate variation in plasticity - The plastic genotype (indicated by the dotted line) induces spines in the predator environment, in contrast to the fixed genotypes that are specialized to low or high predation environments (solid gray or black lines, respectively). The difference in spine length between the plastic genotype and the fixed genotype represents a limit to plasticity. Panel (b) shows the fitness of each genotype (e.g., offspring number, lifespan, adult body size). Adaptive plasticity should translate into maintenance of relatively high fitness across an environmental gradient (as shown here). A cost of the ability to be plastic is represented by lower fitness of the plastic genotype (dotted line) relative to the specialist genotype in the environment where the plastic trait is not expressed (solid lines). While there is a cost of the induced phenotype itself, the benefits of plasticity result in a net fitness benefit relative to the spine-less specialist in the high predation environment. However, any developmental or genetic limits to plastic spine production should result in relatively lower fitness of the plastic genotype when compared to the specialist spine producer. While this example shows reaction norms as genotypes, the same approach can be used for individuals, populations, or species. 
empirical findings have challenged our conceptual understanding. Finally, we discuss exciting areas of future research. Throughout this chapter, we also consider the parallel literature on variation in niche breadth, as in many cases, variation in the degree of generalization is due at least in part to underlying plasticity.

\subsection{SELECTION FOR PLASTICITY: ENVIRONMENTAL VARIATION AND ENVIRONMENTAL EXTREMES}

We first review the conditions expected to favor the evolution of plasticity: variable or novel environments. Experiments in both the lab and field provide support linking the degree of environmental variability to plasticity, but also show that plasticity does not always evolve when expected, pointing to the importance of costs and limits. We often treat characteristics of the environment, such as variability and cue reliability, as external to the organism. However, organismal traits such a dispersal and diapause influence these selective drivers of plasticity, resulting in opportunities for complex feedbacks in the evolution of plasticity.

\subsubsection{Theory Emphasizes the Importance of Environmental Variation}

Phenotypic plasticity is generally favored when the environment varies and different phenotypes are optimal in different environments, especially when costs of phenotype adjustments are low and predictive environmental cues are present (Scheiner 1993; Schlichting and Pigliucci 1998; Berrigan and Scheiner 2004; Scheiner 2020). However, the time scale, or temporal graininess of environmental variation, matters (Figure 6.2). Developmental plasticity is particularly favored when the environment changes across generations, but is relatively consistent within generations ('coarsegrained variation,' Levins 1968; Van Tienderen 1991; Moran 1992). When the environment changes within a generation, more continuous phenotypic adjustments are favored, such as context-dependent expression of behaviors or enzymes ('fine-grained variation,' Bradshaw 1965; Piersma and Drent 2003; Snell-Rood 2013). When conditions change over longer time frames but are consistent across successive generations, transgenerational plasticity can be adaptive (Figure 6.2; Day and Bonduriansky 2011; Bell and Hellmann 2019; see also Bonduriansky 2021 in this volume). While variation tends to favor plasticity, extreme variation can disfavor such strategies. Highly variable environments, especially when reliable cues are lacking, result in the evolution of bet-hedging (Tufto 2015) such as stochastic gene expression in microbes (Veening et al. 2008) or dormancy periods in desert annuals (Gremer and Venable 2014).

Theoretical models show that both temporal and spatial environmental variation can play a role in the evolution of plasticity (Berrigan and Scheiner 2004). Plasticity is usually favored in the face of temporal variation with reliable cues, but specialization may emerge in spatially variable conditions if one environment is more common or if patterns of selection across environments are very different (Van Tienderen 1991; Scheiner 1993). As dispersal between spatially variable environments increases, population-level selection on plasticity increases; when migration rates are lower, spatial variation results in a relative increase in the strength of local selection (Scheiner 1998). Models that integrate both spatial and temporal variation suggest that spatial variation can be a 


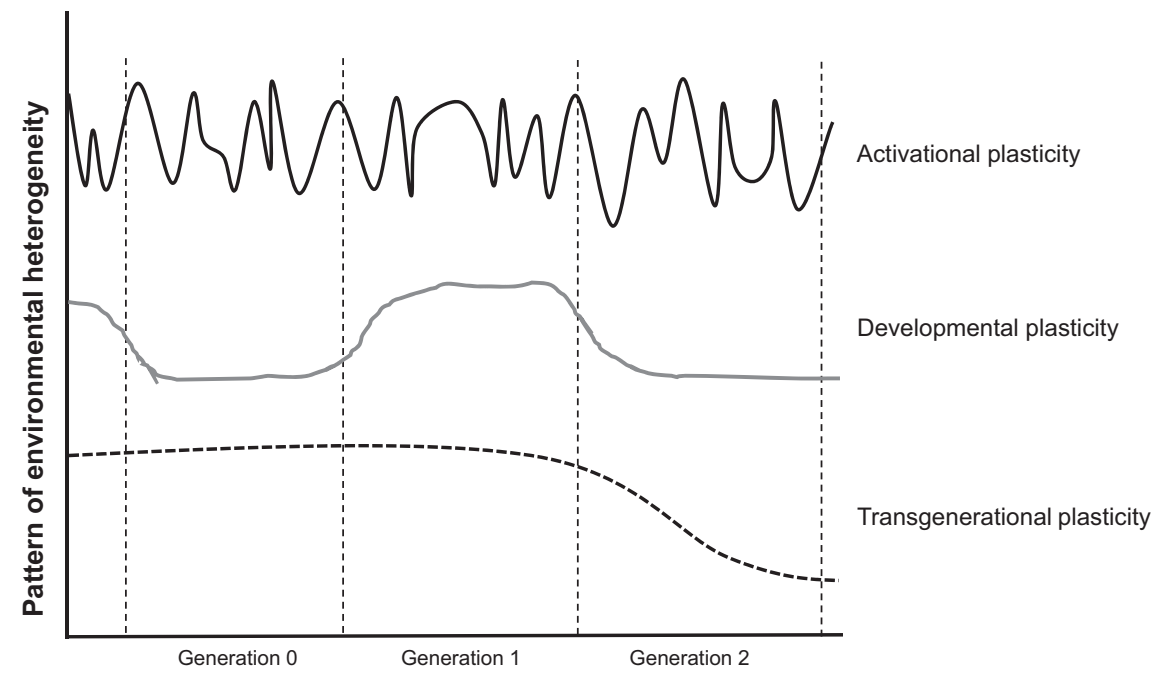

FIGURE 6.2 Patterns of environmental variability and forms of plasticity. The environment can vary over space or time. Fine-grained environmental variation (top) refers to environmental variation within a generation and favors the evolution of 'activational' plasticity (the activation of underlying pre-existing networks, such as behavior or physiology) or forms of developmental plasticity that are reversible. Fine-grained variation can also select for fixed average traits or bet-hedging if the rate of change is rapid, unpredictable, or 'cue-less.' Coarsegrained variation refers to environmental change where environments are relatively constant within generations. Such variation can be due to relatively slower environmental change, or organismal traits such as habitat choice that increase environmental constancy within generations. This form of variation tends to favor developmental plasticity, where early cues on the state of the environment trigger different developmental trajectories, which tend to have broader, more integrated phenotypic effects that are often less reversible. Finally, when rates of environmental variation are very slow across generations, there may be selection for transgenerational plasticity, where parental cues or conditions shape the development of offspring.

more potent driver of plasticity, but how they combine depends on the life history of the organism (Scheiner 2013). Across these models, theory shows that environmental variation tends to favor the evolution of plasticity, but also combines with other factors, such as costs of plasticity and cue reliability, as we discuss more below.

\subsubsection{Empirical Data Suggests Environmental Variation Is Important, but Not Entirely Predictive}

Empirical studies support the prediction that plasticity should be higher in genotypes that experience greater environmental variation. Genetic variation in plasticity has been linked to the degree of environmental variation in both abiotic and biotic factors (e.g., Van Kleunen and Fischer 2001; Baythavong 2011). Species with higher dispersal rates, which tend to experience greater spatial variation also tend to show greater developmental plasticity (Hollander 2008). Similarly, species with greater 
diet breadth often exhibit greater behavioral plasticity (Hoedjes et al. 2011). While environmental variation often correlates with variation in plasticity across species and populations, in some cases it explains little to no variation in plasticity (Karban and Nagasaka 2004), reinforcing the idea that environmental variation is only a piece of the equation in the evolution of plasticity.

Experimental evolution studies also provide empirical support for the expectation that environmental variation favors the evolution of plastic generalists (Kassen 2002). Some experimental evolution approaches suggest that the evolution of plasticity may be more likely in the face of temporal, rather than spatial, variation (Reboud and Bell 1997; Condon et al. 2014), and the time scale of that variation (fine- or coarsegrained) may matter less than expected (Kassen and Bell 1998). While environmental variation tends to favor the evolution of plasticity in the lab, there are just as many cases where predicted patterns of plasticity and wider niche breadth do not evolve in variable environments (Riddle et al. 1986; Bell 1997; Jasmin and Kassen 2007), suggesting the importance of costs and limits in the evolution of plasticity.

\subsubsection{Evolution of Plasticity as a Byproduct of Strong Directional Selection}

Much of the literature has stressed the importance of environmental variation as a driver of the evolution of plasticity. However, selection in novel or extreme environments, regardless of current levels of environmental variation, can also result in the evolution of plasticity as a byproduct (but see Scheiner and Levis 2021 in this volume). Theory suggests that in extreme environments, outside of the range of recent environmental variation, there may be selection on plasticity as it shifts populations to the new fitness peak (Lande 2009; Chevin and Lande 2010; Chevin et al. 2013). This is similar to other models suggesting that selection away from the population mean is more likely to result in the evolution of plasticity (Gavrilets and Scheiner 1993). Such theory has been offered as an explanation for why experimental evolution sometimes results in an increase in plasticity or phenotypic variance (e.g., Falconer 1990; Czesak et al. 2006; Garland and Kelly 2006). Similarly, artificial selection close to ancestral mean phenotypes results in the evolution of specialists, while selection in more extreme novel environments results in plastic generalists (Hughes et al. 2007). Outside of the lab, extreme novel environments (such as cities) select for behavioral plasticity through both species sorting (Sol et al. 2008; Maklakov et al. 2011) and population differentiation within species (Snell-Rood and Wick 2013). Across these cases, plasticity emerges in more extreme environments but not necessarily in more variable environments. Thus, understanding the evolution of plasticity requires considering not only current patterns of environmental variation but also the difference between ancestral and current conditions.

\subsubsection{Organismal Traits Structure Environmental Variation}

Predictions about the evolution of plasticity often treat environmental variation as a force external to an individual organism. Some of the disconnect between theoretical expectations and diverse empirical findings likely stems from the fact 
that organisms choose and modify their environment such that our measures of environmental variation are poor measures of the variation organisms are actually experiencing (Sultan 2015, Snell-Rood and Steck 2019). Behavioral and physiological traits that influence how organisms experience environmental variation can themselves evolve, setting the stage for complex evolutionary feedbacks (Kylafis and Loreau 2008; Scheiner 2016). In some cases, individual behavior may increase environmental variation, such as neophilic or exploratory behaviors (Greenberg 1990; Tebbich et al. 2009), which could result in positive feedback in the evolution of plasticity, until associated costs take effect. More commonly, individual behavior tends to decrease experienced environmental variation such that an otherwise complex or variable environment is more stable or predictable. For instance, dispersal behavior, coupled with habitat preferences, reduces habitat heterogeneity experienced by an organism (Kotliar and Wiens 1990), and diapause or microhabitat selection can buffer temperature variation (Huey et al. 2003). In many cases, individuals choose habitats based on their own phenotypes or previous experiences (Davis 2008; Edelaar and Bolnick 2012). Such habitat selection or modification tends to reduce environmental variation and drive the evolution of specialization (Ravigne et al. 2009). Combining behaviors that influence environmental variation with important details of life histories, dispersal, and natural history offer more informed predictions for the evolution of plasticity (Scheiner et al. 2012; Scheiner 2016).

\subsubsection{Selection on Plasticity Is Limited by Cue Reliability}

Environmental variation tends to set the stage for the evolution of plasticity. However, plasticity is favored in variable environments only when there are reliable cues about the state of the environment (Moran 1992; DeWitt et al. 1998). In other words, plasticity should evolve when future environmental states vary and cues received in the present provide some reliable information about environmental states in the future (McLinn and Stephens 2006; Botero et al. 2015). If this condition is not satisfied, plastic organisms risk expressing phenotypes that are poorly matched to their environments. Thus, a major limit to the evolution of phenotypic plasticity is the degree to which available cues are reliably predictive of future environmental states.

However, just as organismal traits structure environmental variation, they also influence cue reliability. Organisms are bombarded with a wealth of information on the state of the environment, but, in an evolutionary sense, they have some degree of 'control' in the specific cues they 'use' in developmental decisions. Organisms may increase their certainty in evaluating the current state of the environment by paying close attention to individual cues (Szpiro and Carrasco 2015; Kurtz et al. 2017) or using a combination of different cues (Munoz and Blumstein 2012). Cue use itself is also evolving, and organisms focus their attention on more reliable cues (Dunlap and Stephens 2014) and attend to these particularly relevant cues during certain developmental windows (Bateson 1979; Fawcett and Frankenhuis 2015). Thus, cue reliability can itself evolve and likely carry associated costs, setting the stage for complex feedbacks in the evolution of plasticity. 


\subsection{COSTS AND LIMITS SELECT AGAINST PLASTICITY, BUT ARE OFTEN LOWER THAN EXPECTED}

Variable and extreme environments can drive the evolution of plasticity, especially if reliable and predictive cues of environmental states are available. We next review the costs and limits that select against plasticity (DeWitt et al. 1998; Callahan et al. 2008; Murren et al. 2015). Interestingly, literature searches suggest that existing research focuses on the costs and limits of plasticity more than twice as often as the drivers of plasticity. 'Costs' represent fitness tradeoffs associated with plasticity, while 'limits' on plasticity stem from situations where the plastic genotypes cannot achieve the same phenotype as fixed genotypes (DeWitt et al. 1998; Auld et al. 2010). For example, in Daphnia, a cost of the ability to induce a spine in response to a predator would be detected as a lower fitness of the plastic genotype in the predator-free environment relative to a specialist that does not express a spine (Figure 6.1). However, a limit to plasticity would be seen as the plastic genotype inducing a less pronounced spine relative to a specialist that produces a spine across all environments. Depending on the traits measured, an experiment may detect fitness costs that stem from a phenotypic limit (Figure 6.1); considering the underlying mechanism of costs and limits can provide insights into the processes influencing the evolution of plasticity.

\subsubsection{Conflict between Theoretical Expectations of Costs and Empirical Observations}

Models of the evolution of plasticity show that costs of plasticity reduce the likelihood of plasticity evolving, often favoring the evolution of a fixed average phenotype or specialization (Scheiner 1993; Schlichting and Pigliucci 1998; Sultan and Spencer 2002; Berrigan and Scheiner 2004). In considering the evolution of plasticity, we must distinguish between costs of an induced plastic phenotype and costs of the ability to be plastic (DeWitt et al 1998; Callahan et al. 2008; Murren et al. 2015). Many traits induced through plasticity are themselves costly, for instance the fitness tradeoffs associated with growing a defensive spine in Daphnia (Black and Dodson 1990), or the costs of upregulating chemical defenses in plants (Cipollini et al. 2014). The trait- or environment-specific costs of plastic or induced phenotypes are rampant. However, costs of the ability to be plastic are thought to be of primary importance in explaining genetic variation in plasticity because: (1) trait-specific costs should also be experienced by fixed genotypes expressing that trait, and (2) trait-specific costs are often offset by the benefits of an induced trait in a particular environment. We focus our discussion on the costs of the ability to be plastic, noting that trait-specific costs can be important limits in the evolution of plasticity when developmental time lags create phenotypic mismatches (Padilla and Adolph 1996).

A large body of empirical work has sought to measure the costs of the ability to be plastic. In some cases, studies have found these expected costs. For instance, in radish, the ability to mount an induced defense in response to predation is costly in terms of lifetime fruit mass (Agrawal et al. 2002). In frogs, the ability to alter developmental timing in response to pool drying is costly in terms of size at metamorphosis, 
at least in some populations (Merila et al. 2004). However, empirical studies often do not find the expected costs of plasticity. For example, there are few to no costs of the ability to induce a defensive spine in Daphnia, despite costs of the spine itself (Scheiner and Berrigan 1998). Similarly, there are weak to absent costs of predatorinduced plasticity in frogs (Steiner and Van Buskirk 2008). Overall, meta-analyses across 27 studies show that while costs of plasticity are detected more often than expected by chance, overall, they are infrequent ( $<30 \%$ of tests for costs) and their effects are relatively small ( $\mid$ selection coefficients $\mid<0.1$, Van Buskirk and Steiner 2009). In addition, costs of plasticity tend to be higher when traits are measured in stressful conditions (Van Buskirk and Steiner 2009). For instance, in mustards, a cost of the ability to adaptively adjust leaf area with light is only seen under low light conditions (Steinger et al. 2003).

The literature on costs and tradeoffs of niche breadth (Futuyma and Moreno 1988; Sexton et al. 2017) provides similarly varied evidence for costs. There is ample evidence for the often-assumed generalist-specialist tradeoff (e.g., Kelly and Bowers 2016; Thuy et al. 2016), but there are often cases where costs are lower than expected, or completely absent (Dutilleul et al. 2017; Fukano and Nakayama 2018). Experimental evolution studies of adaptation to alternate environments overwhelmingly show tradeoffs in fitness between environments, but detecting costs of simultaneous adaptation to both environments is less likely (Kassen 2002). We next explore the mechanisms that may generate costs, and explanations for why costs may be less common than expected.

\subsubsection{When We See Costs, Where Are They Coming From?}

Fitness tradeoffs of plasticity and wide niche breadth can come from a number of underlying mechanisms (DeWitt et al. 1998; Auld et al. 2010). Costs of plasticity refer to fitness tradeoffs of the ability to be plastic (Figure 6.1), and some of the best examples stem from developmental costs of plasticity itself. Plastic genotypes, relative to specialist genotypes, must sense cues about the state of the environment, process those cues, and develop the appropriate matched phenotype. Regardless of the actual phenotype, this process can be costly, especially for forms of plasticity that develop through learning-like processes ('developmental selection,' Snell-Rood et al 2018). For instance, generalist insects that use a range of host plants must attend to a wider range of cues than specialists, which takes time and divides attention thereby potentially increasing the risk of predation (Bernays 2001; Dukas 2002). Organisms that adjust their behavior through learning must spend time and energy sampling the environment and consolidating information (Dukas 1998; Dukas 2019). The associated neural machinery and process of forming long-term memories are metabolically costly (Laughlin et al. 1998; Mery and Kawecki 2005), and large brains result in exponential increases in developmental time (Workman et al. 2013). Thus, the evolution of learning results in tradeoffs in juvenile competitive ability (Mery and Kawecki 2003) and adult reproduction, often delaying reproduction and resulting in greater investment in fewer offspring (Barrickman et al. 2008; Snell-Rood et al. 2011; Kotrschal et al. 2013). In many 
cases, there are even direct tissue tradeoffs between plasticity machinery (such as brain size) and other costly tissues, such as gut or flight muscle (Isler and van Schaik 2006; Liao et al. 2016). Similar costs of the process of plasticity are seen for other types of plasticity that develop through variation and selection within individuals such as acquired immunity or plant morphological architecture (SnellRood et al. 2018).

Limits to plasticity stem from constraints on plastic genotypes expressing optimal phenotypes relative to a specialist. These limits may be measured in terms of fitness costs, but these costs of 'phenotypic inferiority' originate from development and genetic constraints. These limits more broadly refer to the idea that 'jack-of-all-trades is master of none.' First, let us review limits that stem from developmental processes. The 'developmental range' limit states that by specializing on a particular fixed trait, non-plastic genotypes may be able to achieve more extreme trait values or those that are better matched to the environment in question (DeWitt et al. 1998). This idea is supported by the observation that the range of plasticity seen within species is often dwarfed by trait divergence across species. For instance, developmental plasticity in relative limb length induced by rearing substrate within Anolis species is much smaller than differences across species of twig- and trunk specialists (Losos et al. 2000). Similarly, activityinduced variation in mouse morphology is much less than genetic variation across lines artificially selected for running activity (Kelly et al. 2006). Another developmental constraint is time lags. The development of well-matched phenotypes often takes time, which may be an issue when the environment itself is changing at rate that is faster than an organism can develop an appropriate phenotype, resulting in a suboptimal phenotype in the current environment (Padilla and Adolph 1996; Gabriel et al. 2005). A final limit to the developmental process is the 'epiphenotype problem,' which posits that the earlier in development that salient cues are received, the greater range of possible resultant phenotypic outcomes (DeWitt et al. 1998). Thus, plastic genotypes may have less integrated and more poorly performing phenotypes than specialists due to limits on the timing of developmental processes. Some empirical support exists for the epiphenotype hypothesis: for instance, in snails that alter shell shape in response to predator cues, those that received cues earlier in development are able to achieve a greater range of shell phenotypes (Hoverman and Relyea 2007). Species of shorebirds and ducks that hatch relatively earlier show greater phenotypic variation within species in relative bill and wing dimensions (Snell-Rood et al. 2015). Limits to developmental processes can result in poorer performance of plastic phenotypes compared to when those phenotypes are relatively more fixed in development.

Limits to plasticity can also arise from evolutionary constraints at the level of the underlying genetic architecture of plasticity. Antagonistic pleiotropy refers to situations where the action of a gene in one situation is negatively correlated with its action in another environment. Experimental evolution studies commonly show evidence of antagonistic pleiotropy when organisms are adapting to heterogeneous environments (Kassen 2002). For instance, flies selected in variable temperature environments are plastic generalists but perform poorly 
in specific temperature conditions relative to evolved specialists (Berger et al. 2014). Tradeoffs resulting from adaption to a particular environment can be more pronounced when considering environments that are relatively more dissimilar (Travisano and Lenski 1996). This observation is consistent with plasticity theory showing that highly divergent environments, with different optimal trait values, favor the evolution of distinct specialists (Levins 1968; Scheiner 1993), resulting in phenotypic divergence across species that dwarfs within-species plasticity (e.g., Losos et al. 2000). Limits to the evolution of plasticity and niche breadth may also arise from the efficacy of selection across environments. Because specialists are adapting to one environment, and selection is spread across multiple environments in generalist species, specialists will fix beneficial mutations and purge deleterious mutations faster for traits specific to individual environments (Kawecki 1994; Whitlock 1996; Van Dyken and Wade 2010, Snell-Rood et al. 2010). Empirical support for this idea comes from experimental evolution studies where specialists adapt more rapidly due to increased efficacy of selection (Bennett et al. 1992; Kassen and Bell 1998) and genes biased in their expression between alternate morphs being more genetically variable, presumably due to relaxed purifying selection (Kijimoto et al. 2014). However, other experiments fail to find support for this idea (Cooper and Lenski 2000), and still others question the theoretical assumption of plastic gene expression being entirely specific to alternate environments (Snell-Rood et al. 2010).

\subsubsection{Why Don't We See Costs of Plasticity More Consistentiy Across Studies?}

As this discussion shows, a diversity of mechanisms can generate fitness tradeoffs associated with plasticity. These explanations are not mutually exclusive, and the mixed evidence for each suggests that they are all important to varying degrees. Indeed, this diversity of mechanisms is one explanation for why the evidence for costs of plasticity and generalization is so varied (Kassen 2002; Van Buskirk and Steiner 2009). It is likely that different types of costs apply differently to plastic traits depending on how they develop and function. Indeed, it has been argued that developmental switch mechanisms of plasticity are more likely to be limited by developmental and genetic limits acting at the population level, while learning-like mechanisms of plasticity are more likely to be limited by costs to the individual of the developmental process itself (Snell-Rood 2012; Snell-Rood et al. 2018). Here we review several additional explanations for why the costs of plasticity tend to be lower and less frequent than often expected. These explanations fall into two general categories: patterns of past selection and how the complexity of organisms both affect our ability to detect costs. This discussion recalls broader discussions around the challenges of detecting costs and tradeoffs (Reznick et al. 2000; Agrawal 2020).

First, patterns of past selection affect our ability to detect costs. There should be strong selection against the costs of plasticity, and over time, mechanisms should evolve to reduce the costs of plasticity (Murren et al. 2015). For example, 
predator-induced spines in Daphnia are themselves costly, resulting in lower fecundity (Riessen and Sprules 1990). However, some Daphnia also reduce their metabolic rate in the face of predation, which can sometimes offset the costs of an induced spine (Scheiner and Berrigan 1998). The idea that the costs of plasticity are evolutionarily transient is similar to the argument that costs of elaborated sexual traits are fleeting as there is strong selection on mechanisms to reduce these costs (Badyaev 2004). For instance, changes in underlying metabolic pathways can affect the costs of acquiring carotenoids and the development of colorful ornaments (McGraw 2005; Higginson et al. 2016). Evolutionary changes that reduce costs can also explain why we sometimes detect costs within species, but such tradeoffs are not seen across species (Agrawal 2020). Evolutionary innovations can explain instances of synergistic pleiotropy, instead of the commonly assumed antagonistic pleiotropy (e.g., Sackman and Rokyta 2019; Ruark-Seward et al. 2020): as genotypes adapt to multiple environments, some mutations are just better than others. From a methodological standpoint, how do we deal with selection altering the relative costs and benefits of plasticity over time? Experimental evolution studies offer one approach to tease apart the effects of time: for instance, costs of specialization take time to emerge in flies adapting to different environments (Olazcuaga et al. 2019). Similarly, one could account for evolutionary time in comparisons of costs across populations or species. Another approach is the use of recombinant inbred lines in the lab; because these lines have not experienced selection in the field, they may better reveal costs of the ability to be plastic (Weinig et al. 2006). Experimentally detecting costs of plasticity also depends on the presence of variation in plasticity-either variation in the degree of plasticity or the presence of specialists and generalists. In many cases, selection on plasticity may be so strong or historically ancient that there is little current variation in the degree of plasticity. For instance, there is no standing genetic variation in adaptive developmental plasticity in some populations of spadefoot toads (Newman 1988). While we may detect such 'genotype-by-environment' interactions (i.e., $G x E$ ) in our analyses, in some cases it could be a function of noise, instead of functional standing variation in a population. Negative correlations between the number of lines in a study and the probability of detecting costs of plasticity support the idea that meaningful variation in $G x E$ may limit the ability to detect costs (Van Buskirk and Steiner 2009).

Second, the complexity of organisms means that it is often difficult to determine the best traits to measure with respect to adaptive plasticity. Many interacting traits, at levels from proteins to behavior, affect the fitness of individual organisms. How does one choose the best traits to measure when studying costs of adaptive plasticity? Meta-analyses show that we are just as likely to detect costs of plasticity as to detect costs of 'canalization;' i.e., keeping a phenotype stable in the face of environmental variation (Van Buskirk and Steiner 2009). Traits closely related to fitness, such as body size and development time, are often under selection to be maintained across an environmental gradient (Van Tienderen 1991). Hence, a cost of canalization may indeed reflect a cost of plasticity, but for a trait not measured. In support of this interpretation, meta-analyses show that studies lack evidence for 
adaptive plasticity in $71 \%$ of cases testing for costs of plasticity (Van Buskirk and Steiner 2009), suggesting in many cases we may be choosing the 'wrong' traits as proxies of plasticity. What does this mean for future studies? There have been a number of calls to quantify biological complexity more thoroughly, from more complete measures of fitness (Shaw et al. 2008) to measures of the diversity of cues triggering plasticity (Westneat et al. 2019). However, we caution that more data does not necessarily give more clarity without informed expectations: given the natural history of a system, which phenotypic traits does one expect to vary to maintain constant fitness-related traits across environments? In expanding the range of traits measured, we may also want to consider what aspects of performance we tend to miss in laboratory assays that could be relevant to costs of plasticity in the field. For instance, in the lab, plastic generalists are sometimes more fecund overall (e.g., Drosophila thermal generalists; Condon et al. 2014), opposite to expectations. It is possible that such genotypes are tolerant of novel environments with few competitors, but intolerant to competition (sensu 'Grimes Triangle'), but we would miss such tradeoffs in lab assays with abundant resources (Agrawal et al. 2010; Cipollini et al. 2014).

\subsection{FUTURE DIRECTIONS IN ECOLOGY AND EVOLUTION OF PLASTICITY}

Given the extensive work to date on the ecological and evolutionary drivers and constraints on plasticity, what do we have left to explore? Increasing attention to environmental change and developmental mechanisms of plasticity are opening new doors for theoretical and empirical advances in our understanding of why plasticity varies.

\subsubsection{Plasticity and Cryptic Genetic Variation in Novel Environments}

The role of plasticity in novel environments has received increased attention in the last two decades as we gain a greater understanding of rapid, human-induced environmental change (Sih et al. 2011; Merila and Hendry 2014; see also Diamond and Martin 2021; Levis and Pfennig 2021; and Scheiner and Levis 2021 in this volume). Theory has established that plasticity may allow organisms to achieve greater fitness in novel environments, allowing for population persistence and further adaptation (Price et al. 2003; Lande 2009; Chevin and Lande 2010). However, we know less about when plasticity in novel environments will fuel subsequent adaptive evolution. Cryptic genetic variation can be seen in laboratory experiments (Gibson and Dworkin 2004), but how does it affect evolution in nature (Ledon-Rettig et al. 2014)?

Microevolutionary models that incorporate development—such as simple regulatory network models - are well-suited to address this issue since they model mechanisms through which hidden variation can accumulate (Hoke et al. 2019). Asymmetries in the degree of variation released in novel environments may be a result of underlying developmental systems that channel genetic and environmental variation along phenotypic dimensions that have been favored in the past (Watson and Szathmary 2016). In an important simulation model, for example, genotypes with plastic gene regulatory networks that were allowed to evolve in variable 
environments showed greater alignment of genetic and mutational variance along the dominant axis of environmental variation compared to fixed genotypes (Draghi and Whitlock 2012). These same plastic genotypes, when challenged with a more extreme novel environment along the same dominant axis, were better phenotypically matched to novel environments. This theoretic result demonstrates that selection for plasticity may result in developmental or regulatory systems that constrain the dominant axes of genetic variation, with important implications for further evolution in novel environments. Indeed, a recent meta-analysis found support for this idea that the 'direction of plasticity' was aligned with the dominant axis of genetic variation in novel environments (Noble et al. 2019). Such approaches pave the way for future research addressing whether certain patterns of past selection harbor greater underlying cryptic genetic variation and how such variation interacts with environmental change.

\subsubsection{Costs of Plasticity and Genetic Assimilation in Novel Environments}

As highlighted in this chapter, decades of effort have been spent searching for the costs of plasticity, and while costs have been found, they are incredibly variable in frequency and magnitude. Are there exciting future research directions on the costs of plasticity, or are they an act of futility in an over-studied area? We argue above that one way forward in studies of costs are studies that consider multiple dimensions of fitness in realistic conditions. A particularly interesting context to study costs is with respect to the importance of plasticity in novel environments. Plasticity is more likely to lead to genetic assimilation in novel environments when those new conditions are constant and plasticity is costly (reviewed in Scheiner and Levis 2021 in this volume). We can test such ideas using extreme but predictable anthropogenic environments (e.g., cities, agricultural monocultures; see also Diamond and Martin 2021 in this volume) and forms of plasticity that are known to be costly (e.g., trial-and-error learning). We can also extrapolate to understudied forms of plasticity that should have important costs and benefits in new conditions (e.g., acquired immunity).

\subsubsection{Feedbacks in the Evolution of Plasticity: When the Environment Evolves}

We know organism physiology and behavior can influence how a genotype experiences environmental variability and the reliability of cues, important drivers of plasticity. When these traits are evolving alongside plasticity, complex dynamics can emerge. To what extent could such negative feedbacks explain the evolution of specialists across lineages, or, conversely, positive feedbacks explain the evolution of plastic generalists and situations where generalists fuel evolutionary diversification (see Levis and Pfennig 2021 in this volume)? Organismal traits such as resource use, diapause, and movement affect the ecological conditions that favor or constrain the evolution of plasticity. Thus, a future direction in understanding plasticity lies at an intersection with the emerging literature on eco-evolutionary feedbacks (Govaert et al. 2019). 


\subsubsection{Evaluating Null Models in the Evolution of Plasticity}

Theory recognizes that neutral processes may explain patterns of variation in plasticity and niche breadth (Via 1993; Hardy et al. 2016; Forister and Jenkins 2017), but most empirical studies focus on adaptive explanations. To what extent does genetic drift shape the distribution of specialists and plastic generalists across the tree of life? Does this matter when making predictions about environmental change (Ghalambor et al. 2007; Snell-Rood et al. 2018)? We should move past our adaptationist biases in our explanations of the causes and consequences of plasticity (see also Futuyma 2021).

\subsubsection{Developmental Realism in Evolutionary Models: Predicting Diversification and the Emergence of Specialists and Generalists}

Incorporating more developmental realism into models changes the dynamics of evolutionary theory (Draghi and Whitlock 2012; Kriegman et al. 2018). As we incorporate more features of development into our conceptual links between genotype and phenotype (e.g., Salazar-Ciudad and Jernvall 2010), we believe the field will have more power to develop theory related to macroevolutionary questions, such as speciation and the diversification of body shapes. To what extent does plasticity play a role in these processes? Reviewing the costs and limits of plasticity suggests that antagonistic pleiotropy could explain why, over long periods of evolutionary time, species with highly divergent traits are favored over 'infinitely plastic generalists' relative to individual specialists, it is not possible for one plastic genotype to develop alternate phenotypes that are high-performing over their lifetime. Models of this process that incorporate more developmental realism could integrate underlying costs and limits and ask questions about the emergence of depressed hybrid fitness and speciation, as selection in divergent environments generates antagonistic pleiotropy.

\subsection{CONCLUSIONS}

Adaptive phenotypic plasticity is favored in novel and variable environments and can underlie broad niche breadth. Plastic generalists can colonize new environments, paving the way for diversification. Costs and limits of plasticity play an important role in maintaining variation in plasticity and promoting the evolution of specialists, for instance in constant conditions or when alternate environments favor drastically different phenotypes. Despite decades of research on the ecology and evolution of plasticity, there are still many exciting and new directions of future research (Box 6.1), especially around developing new theory and testing theoretical predictions in the field. Anthropogenic environments such as cities offer a promising context where we can test plasticity theory while also generating insights with conservation implications. Finally, as we expand our conceptualization of the genotype to phenotype link from a simple view of a gene to trait mappings, to more complex network-based interactions, we generate novel insights especially with respect to broader macroevolutionary questions. 


\section{BOX 6.1 SUGGESTIONS FOR FUTURE RESEARCH: QUESTIONS AND HYPOTHESES}

- When does plasticity result in adaptive traits in novel environments? Developmental selection mechanisms of plasticity are more likely to result in adaptive plasticity in novel environments (relative to evolved switches).

- How does plasticity interact with cryptic genetic variation in novel environments? Patterns of past selection bias underlying patterns of genetic variation (e.g., along the same axes of environmental variation).

- Why do studies vary so much in the likelihood of detecting costs of plasticity? Costs of plasticity play out across multiple dimensions of fitness and are often obscured in laboratory conditions.

- When do costs of plasticity shape the ecology and evolution of plasticity? Costs of plasticity may play a prominent but transient role in genetic assimilation in novel environments.

- Why does plasticity vary within and between species? Neutral theories can explain patterns of plasticity, in some cases, better than adaptive explanations.

- How does plasticity bridge the divide between micro- and macroevolutionary questions? Developmental realism in evolutionary models could generate predictions around the emergence of speciation and specialization as developmental systems adapt to different environments.

\section{REFERENCES}

Agrawal, A. A. 2020. A scale-dependent framework for trade-offs, syndromes, and specialization in organismal biology. Ecology 101(2):e02924.

Agrawal, A. A., J. K. Conner, M. T. J. Johnson, and R. Wallsgrove. 2002. Ecological genetics of an induced plant defense against herbivores: Additive genetic variance and costs of phenotypic plasticity. Evolution 56:2206-2213.

Agrawal, A. A., J. K. Conner, and S. Rasmann. 2010. Tradeoffs and negative correlations in evolutionary ecology, pp. 243-268. In M. A. Bell, D. J. Futuyma, W. F. Eanes, and J. S. Levinton, eds., Evolution since Darwin: The First 150 Years. Sinauer Associates, Sunderland, Massachusetts.

Auld, J. R., A. A. Agrawal, and R. A. Relyea. 2010. Re-evaluating the costs and limits of adaptive phenotypic plasticity. Proceedings of the Royal Society B-Biological Sciences 277:503-511.

Badyaev, A. 2004. Integration and modularity in the evolution of sexual ornaments: An overlooked perspective, pp. 50-79. In M. Pigluicci and K. Preston, eds., Phenotypic Integration: Studying the Ecology and Evolution of Complex Phenotypes. Oxford University Press, Oxford. 
Barrickman, N. L., M. L. Bastian, K. Isler, and C. P. van Schaik. 2008. Life history costs and benefits of encephalization: A comparative test using data from long-term studies of primates in the wild. Journal of Human Evolution 54:568-590.

Bateson, P. 1979. How do sensitive periods arise and what are they for? Animal Behaviour 27:470-486.

Baythavong, B. S. 2011. Linking the spatial scale of environmental variation and the evolution of phenotypic plasticity: Selection favors adaptive plasticity in fine-grained environments. American Naturalist 178:75-87.

Bell, G. A. C. 1997. Experimental evolution in Chlamydomonas.1. Short-term selection in uniform and diverse environments. Heredity 78:490-497.

Bell, A. M. and J. K. Hellmann. 2019. An integrative framework for understanding the mechanisms and multigenerational consequences of transgenerational plasticity. Annual Review of Ecology, Evolution, and Systematics 50:97-118.

Bennett, A. F., R. E. Lenski, and J. E. Mittler. 1992. Evolutionary adaptation to temperature 1. Fitness responses of Escherichia coli to chandes in its thermal environment. Evolution 46:16-30.

Berger, D., R. J. Walters and W. U. Blanckenhorn. 2014. Experimental evolution for generalists and specialists reveals multivariate genetic constraints on thermal reaction norms. Journal of Evolutionary Biology 27:1975-1989.

Bernays, E. A. 2001. Neural limitations in phytophagous insects: Implications for diet breadth and evolution of host affiliation. Annual Review of Entomology 46:703-727.

Berrigan, D. and S. M. Scheiner. 2004. Modeling the evolution of phenotypic plasticity, pp. 82-97. In T. J. DeWitt and S. M. Scheiner, eds., Phenotypic Plasticity: Functional and Conceptual Approaches. Oxford University Press, Oxford.

Black, A. R. and S. I. Dodson. 1990. Demographic costs of Chaoborus-induced phenotypic plasticity in Daphnia pulex. Oecologia 83:117-122.

Bonduriansky, R. 2021. Plasticity across generations. In D. W. Pfennig, ed., Phenotypic Plasticity and Evolution: Causes, Consequences, Controversies. CRC Press, Boca Raton, FL, pp. 327-348.

Botero, C. A., F. J. Weissing, J. Wright, and D. R. Rubenstein. 2015. Evolutionary tipping points in the capacity to adapt to environmental change. Proceedings of the National Academy of Sciences of the United States of America 112:184-189.

Bradshaw, A. D. 1965. Evolutionary significance of phenotypic plasticity in plants. Advances in Genetics 13:115-155.

Callahan, H. S., H. Maughan, and U. K. Steiner. 2008. Phenotypic plasticity, costs of phenotypes, and costs of plasticity toward an integrative view. Evolutionary Biology 1133:44-66.

Chevin, L. M., R. Gallet, R. Gomulkiewicz, R. D. Holt, and S. Fellous. 2013. Phenotypic plasticity in evolutionary rescue experiments. Philosophical Transactions of the Royal Society B-Biological Sciences 268:20120089.

Chevin, L.-M. and R. Lande. 2010. When do adaptive plasticity and genetic evolution prevent extinction of a density regulated population? Evolution 64:1143-1150.

Cipollini, D., D. Walters, and C. Voelckel. 2014. Costs of resistance in plants: From theory to evidence. Insect-Plant Interactions 47:263-307.

Condon, C., B. S. Cooper, S. Yeaman, and M. J. Angilletta, Jr. 2014. Temporal variation favors the evolution of generalists in experimental populations of Drosophila melanogaster. Evolution 68:720-728.

Cooper, V. S. and R. E. Lenski. 2000. The population genetics of ecological specialization in evolving Escherichia coli populations. Nature 407:736-739.

Czesak, M. E., C. W. Fox, and J. B. Wolf. 2006. Experimental evolution of phenotypic plasticity: How predictive are cross-environment genetic correlations? American Naturalist 168:323-335. 
Davis, J. M. 2008. Patterns of variation in the influence of natal experience on habitat choice. Quarterly Review of Biology 83:363-380.

Day, T. and R. Bonduriansky. 2011. A unified approach to the evolutionary consequences of genetic and nongenetic inheritance. American Naturalist 178:e18-e36.

DeWitt, T. J., A. Sih, and D. S. Wilson. 1998. Costs and limits of phenotypic plasticity. Trends in Ecology \& Evolution 13:77-81.

Diamond, S. E. and R. A. Martin. 2021. Buying time: Plasticity and population persistence. In D. W. Pfennig, ed., Phenotypic Plasticity and Evolution: Causes, Consequences, Controversies. CRC Press, Boca Raton, FL.

Draghi, J. A. and M. C. Whitlock. 2012. Phenotypic plasticity facilitates mutational variance, genetic variance and evolvability along the major axis of environmental variation. Evolution 66(9):2891-2902.

Dukas, R. 1998. Evolutionary ecology of learning, pp. 129-174. In R. Dukas, ed., Cognitive Ecology: The Evolutionary Ecology of Information Processing and Decision Making. University of Chicago Press, Chicago, IL.

Dukas, R. 2002. Behavioural and ecological consequences of limited attention. Philosophical Transactions of the Royal Society B-Biological Sciences 357:1539-1547.

Dukas, R. 2019. Animal expertise: Mechanisms, ecology and evolution. Animal Behaviour 147:199-210.

Dunlap, A. S. and D. W. Stephens. 2014. Experimental evolution of prepared learning. Proceedings of the National Academy of Sciences of the United States of America 111:11750-11755.

Dutilleul, M., D. Reale, B. Goussen, C. Lecomte, S. Galas, and J. M. Bonzom. 2017. Adaptation costs to constant and alternating polluted environments. Evolutionary Applications 10:839-851.

Edelaar, P. and D. I. Bolnick. 2012. Non-random gene flow: An underappreciated force in evolution and ecology. Trends in Ecology \& Evolution 27:659-665.

Falconer, D. S. 1990. Selection in different environments: Effects on environmental sensitivity (reaction norm) and on mean performance. Genetical Research 56:57-70.

Fawcett, T. W. and W. E. Frankenhuis. 2015. Adaptive explanations for sensitive windows in development. Frontiers in Zoology 12:S3.

Forister, M. L. and S. H. Jenkins. 2017. A neutral model for the evolution of diet breadth. American Naturalist 190:E40-E54.

Fukano, Y. and S. Nakayama. 2018. An experimental test of trade-offs associated with the adaptation to alternate host plants in the introduced herbivorous beetle, Ophraella communa. Journal of Insect Behavior 31:490-502.

Futuyma, D. J. 2021. How does phenotypic plasticity fit into evolutionary theory? In D. W. Pfennig, ed., Phenotypic Plasticity and Evolution: Causes, Consequences, Controversies. CRC Press, Boca Raton, FL.

Futuyma, D. J. and G. Moreno. 1988. The evolution of ecological specialization. Annual Review of Ecology and Systematics 19:207-233.

Gabriel, W., B. Luttbeg, A. Sih, and R. Tollrian. 2005. Environmental tolerance, heterogeneity, and the evolution of reversible plastic responses. American Naturalist 166:339-353.

Garland, T., Jr. and S. A. Kelly. 2006. Phenotypic plasticity and experimental evolution. Journal of Experimental Biology 209:2344-2361.

Gavrilets, S. and S. M. Scheiner. 1993. The genetics of phenotypic plasticity 6 . Theoretical predictions for directional selection. Journal of Evolutionary Biology 6:49-68.

Ghalambor, C. K., J. K. McKay, S. P. Carrol, and D. N. Reznick. 2007. Adaptive versus nonadaptive phenotypic plasticity and the potential for contemporary adaptation in new environments. Functional Ecology 21:394-407.

Gibson, G. and I. Dworkin. 2004. Uncovering cryptic genetic variation. Nature Reviews Genetics 5:681-611. 
Gilbert, S. F. and D. Epel. 2015. Ecological Developmental Biology. Oxford University Press, Oxford.

Govaert, L., E. A. Fronhofer, S. Lion, C. Eizaguirre, D. Bonte, M. Egas, A. P. Hendry, A. D. Martins, C. J. Melian, J. A. M. Raeymaekers, Ratikainen, II, B. E. Saether, J. A. Schweitzer, and B. Matthews. 2019. Eco-evolutionary feedbacks-theoretical models and perspectives. Functional Ecology 33:13-30.

Greenberg, R. 1990. Feeding neophobia and ecological plasticity: A test of the hypothesis with captive sparrows. Animal Behaviour 39:375-379.

Gremer, J. R. and D. L. Venable. 2014. Bet hedging in desert winter annual plants: optimal germination strategies in a variable environment. Ecology Letters 17:380-387.

Hardy, N. B., D. A. Peterson, and B. B. Normark. 2016. Nonadaptive radiation: Pervasive diet specialization by drift in scale insects? Evolution 70:2421-2428.

Higginson, D. M., V. Belloni, S. N. Davis, E. S. Morrison, J. E. Andrews, and A. V. Badyaev. 2016. Evolution of long-term coloration trends with biochemically unstable ingredients. Proceedings of the Royal Society B-Biological Sciences 283:20160403.

Hoedjes, K. M., H. M. Kruidhof, M. E. Huigens, M. Dicke, L. E. M. Vet, and H. M. Smid. 2011. Natural variation in learning rate and memory dynamics in parasitoid wasps: Opportunities for converging ecology and neuroscience. Proceedings of the Royal Society B-Biological Sciences 278:889-897.

Hoke, K. L., E. Adkins-Regan, A. H. Bass, A. R. McCune, and M. F. Wolfner. 2019. Co-opting evo-devo concepts for new insights into mechanisms of behavioural diversity. Journal of Experimental Biology 222(8):jeb190058.

Hollander, J. 2008. Testing the grain-size model for the evolution of phenotypic plasticity. Evolution 62:1381-1389.

Hoverman, J. T. and R. A. Relyea. 2007. How flexible is phenotypic plasticity? Developmental windows for trait induction and reversal. Ecology 88:693-705.

Huey, R. B., P. E. Hertz, and B. Sinervo. 2003. Behavioral drive versus behavioral inertia in evolution: A null model approach. American Naturalist 161:357-366.

Hughes, B. S., A. J. Cullum, and A. F. Bennett. 2007. Evolutionary adaptation to environmental pH in experimental lineages of Escherichia coli. Evolution 61:1725-1734.

Isler, K. and C. van Schaik. 2006. Costs of encephalization: the energy trade-off hypothesis tested on birds. Journal of Human Evolution 51:228-243.

Jasmin, J. N. and R. Kassen. 2007. Evolution of a single niche specialist in variable environments. Proceedings of the Royal Society B-Biological Sciences 274:2761-2767.

Karban, R. and K. Nagasaka. 2004. Are defenses of wild radish populations well matched with variability and predictablity of herbivory? Evolutionary Ecology 18:283-301.

Kassen, R. 2002. The experimental evolution of specialists, generalists, and the maintenance of diversity. Journal of Evolutionary Biology 15:173-190.

Kassen, B. and G. Bell. 1998. Experimental evolution in Chlamydomonas. IV. Selection in environments that vary through time at different scales. Heredity 80:732-741.

Kawecki, T. J. 1994. Accumulation of deleterious mutations and the evolutionary cost of being a generalist. American Naturalist 144:833-838.

Kelly, C. A. and M. D. Bowers. 2016. Preference and performance of generalist and specialist herbivores on chemically defended host plants. Ecological Entomology 41:308-316.

Kelly, S. A., P. P. Czech, J. T. Wight, K. M. Blank, and T. Garland. 2006. Experimental evolution and phenotypic plasticity of hindlimb bones in high-activity house mice. Journal of Morphology 267:360-374.

Kijimoto, T., E. C. Snell-Rood, M. H. Pespeni, G. Rocha, K. Kafadar, and A. P. Moczek. 2014. The nutritionally responsive transcriptome of the polyphenic beetle Onthophagus taurus and the importance of sexual dimorphism and body region. Proceedings of the Royal Society B-Biological Sciences 281:20142084. 
Kotliar, N. B. and J. A. Wiens. 1990. Multiple scales of patchiness and patch structure: A hierarchical framework for the study of heterogeneity. Oikos 59:253-260.

Kotrschal, A., B. Rogell, A. Bundsen, B. Svensson, S. Zajitschek, I. Brannstrom, S. Immler, A. A. Maklakov, and N. Kolm. 2013. Artificial selection on relative brain size in the guppy reveals costs and benefits of evolving a larger brain. Current Biology 23:168-171.

Kriegman, S., N. Cheney, and J. Bongard. 2018. How morphological development can guide evolution. Scientific Reports 8:16057.

Kurtz, P., K. A. Shapcott, J. Kaiser, J. T. Schmiedt, and M. C. Schmid. 2017. The influence of endogenous and exogenous spatial attention on decision confidence. Scientific Reports 7:6431.

Kylafis, G. and M. Loreau. 2008. Ecological and evolutionary consequences of niche construction for its agent. Ecology Letters 11:1072-1081.

Lande, R. 2009. Adaptation to an extraordinary environment by evolution of phenotypic plasticity and genetic assimilation. Journal of Evolutionary Biology 22:1435-1446.

Laughlin, S. B., R. R. D. van Steveninck, and J. C. Anderson. 1998. The metabolic cost of neural information. Nature Neuroscience 1:36-41.

Ledon-Rettig, C. C., D. W. Pfennig, A. J. Chunco, and I. Dworkin. 2014. Cryptic genetic variation in natural populations: A predictive framework. Integrative and Comparative Biology 54:783-793.

Levins, R. 1968. Evolution in Changing Environments: Some Theoretical Explorations. Princeton University Press, Princeton, NJ.

Levis, N. A. and D. W. Pfennig. 2021. Innovation and diversification via plasticity-led evolution. In D. W. Pfennig, ed., Phenotypic Plasticity and Evolution: Causes, Consequences, Controversies. CRC Press, Boca Raton, FL.

Liao, W. B., S. L. Lou, Y. Zeng, and A. Kotrschal. 2016. Large brains, small guts: The expensive tissue hypothesis supported within anurans. American Naturalist 188:693-700.

Losos, J. B., D. A. Creer, D. Glossip, R. Goellner, A. Hampton, G. Roberts, N. Haskell, P. Taylor, and J. Ettling. 2000. Evolutionary implications of phenotypic plasticity in the hindlimb of the lizard Anolis sagrei. Evolution 54:301-305.

Maklakov, A. A., S. Immler, A. Gonzalez-Voyer, J. Ronn, and N. Kolm. 2011. Brains and the city: Big-brained passerine birds succeed in urban environments. Biology Letters 7:730-732.

McGraw, K. J. 2005. Interspecific variation in dietary carotenoid assimilation in birds: Links to phylogeny and color ornamentation. Comparative Biochemistry and Physiology B-Biochemistry \& Molecular Biology 142:245-250.

McLinn, C. M. and D. W. Stephens. 2006. What makes information valuable: Signal reliability and environmental uncertainty. Animal Behaviour 71:1119-1129.

Merila, J. and A. P. Hendry. 2014. Climate change, adaptation, and phenotypic plasticity: The problem and the evidence. Evolutionary Applications 7:1-14.

Merila, J., A. Laurila, and B. Lindgren. 2004. Variation in the degree and costs of adaptive phenotypic plasticity among Rana temporaria populations. Journal of Evolutionary Biology 17:1132-1140.

Mery, F. and T. J. Kawecki. 2003. A fitness cost of learning ability in Drosophila melanogaster. Proceedings of the Royal Society of London Series B-Biological Sciences 270:2465-2469.

Mery, F. and T. J. Kawecki. 2005. A cost of long-term memory in Drosophila. Science 308:1148.

Moran, N. A. 1992. The evolutionary maintenance of alternative phenotypes. American Naturalist 139:971-989.

Munoz, N. E. and D. T. Blumstein. 2012. Multisensory perception in uncertain environments. Behavioral Ecology 23:457-462. 
Murren, C. J., J. R. Auld, H. Callahan, C. K. Ghalambor, C. A. Handelsman, M. A. Heskel, J. G. Kingsolver, H. J. Maclean, J. Masel, H. Maughan, D. W. Pfennig, R. A. Relyea, S. Seiter, E. Snell-Rood, U. K. Steiner, and C. D. Schlichting. 2015. Constraints on the evolution of phenotypic plasticity: Limits and costs of phenotype and plasticity. Heredity 115:293-301.

Newman, R. A. 1988. Adaptive plasticity in development of Scaphiopus couchii tadpoles in desert ponds. Evolution 42:774-783.

Noble, D. W. A., R. Radersma, and T. Uller. 2019. Plastic responses to novel environments are biased towards phenotype dimensions with high additive genetic variation. Proceedings of the National Academy of Sciences of the United States of America 116:13452-13461.

Olazcuaga, L., N. O. Rode, J. Foucaud, M. Gautier, C. Deschamps, A. Loiseau, N. Lemenager, B. Facon, V. Ravigne, and R. A. Hufbauer. 2019. Evolution of trade-offs across environments following experimental evolution of the generalist Drosophila suzukii to different fruit media. bioRxiv:749945.

Padilla, D. K. and S. C. Adolph. 1996. Plastic inducible morphologies are not always adaptive: The importance of time delays in a stochastic environment. Evolutionary Ecology 10:105-117.

Pfennig, D. W. 2021. Key questions about phenotypic plasticity. In D. W. Pfennig ed., Phenotypic Plasticity and Evolution: Causes, Consequences, Controversies. CRC Press, Boca Raton, FL.

Pfennig, D. W., M. A. Wund, E. C. Snell-Rood, T. Cruickshank, C. D. Schlichting, and A. P. Moczek. 2010. Phenotypic plasticity's impacts on diversification and speciation. Trends in Ecology \& Evolution 25:459-467.

Piersma, T. and J. Drent. 2003. Phenotypic flexibility and the evolution of organismal design. Trends in Ecology \& Evolution 18:228-233.

Price, T. D., A. Qvarnström, and D. E. Irwin. 2003. The role of phenotypic plasticity in driving genetic evolution. Proceedings of the Royal Society of London, B 270:1433-1440.

Ravigne, V., U. Dieckmann, and I. Olivieri. 2009. Live where you thrive: Joint evolution of habitat choice and local adaptation facilitates specialization and promotes diversity. American Naturalist 174:E141-E169.

Reboud, X. and G. Bell. 1997. Experimental evolution in Chlamydomonas 3. Evolution of specialist and generalist types in environments that vary in space and time. Heredity 78:507-514.

Reznick, D., L. Nunney, and A. Tessier. 2000. Big houses, big cars, superfleas and the costs of reproduction. Trends in Ecology \& Evolution 15:421-425.

Richards, C. L., O. Bossdorf, N. Z. Muth, J. Gurevitch, and M. Pigliucci. 2006. Jack of all trades, master of some? On the role of phenotypic plasticity in plant invasions. Ecology Letters 9:981-993.

Riddle, R. A., P. S. Dawson, and D. F. Zirkle. 1986. An experimental test of the relationship between genetic variation and environmental variation in Tribolium flour beetles. Genetics 113:391-404.

Riessen, H. P. and W. G. Sprules. 1990. Demographic costs of antipredator defenses in Daphnia pulex. Ecology 71:1536-1546.

Ruark-Seward, C. L., B. Bonville, G. Kennedy, and D. A. Rasmussen. 2020. Evolutionary dynamics of Tomato spotted wilt virus within and between alternate plant hosts and thrips. bioRxiv.

Sackman, A. M. and D. R. Rokyta. 2019. No cost of complexity in bacteriophages adapting to a complex environment. Genetics 212:267-276.

Salazar-Ciudad, I. and J. Jernvall. 2010. A computational model of teeth and the developmental origins of morphological variation. Nature 464:583-U138.

Scheiner, S. M. 1993. Genetics and evolution of phenotypic plasticity. Annual Review of Ecology and Systematics 24:35-68. 
Scheiner, S. M. 1998. The genetics of phenotypic plasticity. VII. Evolution in a spatiallystructured environment. Journal of Evolutionary Biology 11:303-320.

Scheiner, S. M. 2013. The genetics of phenotypic plasticity. XII. Temporal and spatial heterogeneity. Ecology and Evolution 3:4596-4609.

Scheiner, S. M. 2016. Habitat choice and temporal variation alter the balance between adaptation by genetic differentiation, a jack-of-all-trades strategy, and phenotypic plasticity. American Naturalist 187:633-646.

Scheiner, S. M. 2020. The theory of the evolution of plasticity, pp. 254-272. In S. M. Scheiner and D. P. Mindell, eds., The Theory of Evolution. University of Chicago Press, Chicago, IL.

Scheiner, S. M. and D. Berrigan. 1998. The genetics of phenotypic plasticity. VIII. The cost of plasticity in Daphnia pulex. Evolution 52:368-378.

Scheiner, S. M. and N. A. Levis. 2021. The loss of phenotypic plasticity via natural selection: genetic assimilation. In D. W. Pfennig, ed., Phenotypic Plasticity and Evolution: Causes, Consequences, Controversies. CRC Press, Boca Raton, FL.

Scheiner, S. M., M. Barfield, and R. D. Holt. 2012. The genetics of phenotypic plasticity. XI. Joint evolution of plasticity and dispersal rate. Ecology and Evolution 2:2027-2039.

Schlichting, C. D. and M. Pigliucci. 1998. Phenotypic Evolution: A Reaction Norm Perspective. Sinauer Associates, Sunderland, MA.

Sexton, J. P., J. Montiel, J. E. Shay, M. R. Stephens, and R. A. Slatyer. 2017. Evolution of ecological niche breadth. Annual Review of Ecology, Evolution, and Systematics, 48:183-206.

Shaw, R. G., C. J. Geyer, S. Wagenius, H. H. Hangelbroek, and J. R. Etterson. 2008. Unifying life-history analyses for inference of fitness and population growth. American Naturalist 172:E35-E47.

Sih, A., M. C. O. Ferrari, and D. J. Harris. 2011. Evolution and behavioural responses to human-induced rapid environmental change. Evolutionary Applications 4:367-387.

Snell-Rood, E. C. 2012. Selective processes in development: Implications for the costs and benefits of phenotypic plasticity. Integrative and Comparative Biology 52:31-42.

Snell-Rood, E. C. 2013. An overview of the evolutionary causes and consequences of behavioural plasticity. Animal Behaviour 85:1004-1011.

Snell-Rood, E. C. and M. K. Steck. 2019. Behaviour shapes environmental variation and selection on learning and plasticity: Review of mechanisms and implications. Animal Behaviour 147:147-156.

Snell-Rood, E. C. and N. Wick. 2013. Anthropogenic environments exert variable selection on cranial capacity in mammals. Proceedings of the Royal Society B-Biological Sciences 280, 20131384.

Snell-Rood, E. C, J. D. Van Dyken, T. Cruickshank, M. Wade, and A. Moczek. 2010. Toward a population genetic framework of developmental evolution: Costs, limits, and consequences of phenotypic plasticity. BioEssays 32:71-81.

Snell-Rood, E. C., G. Davidowitz, and D. R. Papaj. 2011. Reproductive tradeoffs of learning in a butterfly. Behavioral Ecology 22:291-302.

Snell-Rood, E. C., E. M. Swanson, and R. L. Young. 2015. Life history as a constraint on plasticity: Developmental timing is correlated with phenotypic variation in birds. Heredity 115:379-388.

Snell-Rood, E. C., M. E. Kobiela, K. L. Sikkink, and A. M. Shephard. 2018. Mechanisms of plastic rescue in novel environments. Annual Review of Ecology, Evolution, and Systematics 49:331-354.

Sol, D., R. P. Duncan, T. M. Blackburn, P. Cassey, and L. Lefebvre. 2005. Big brains, enhanced cognition, and response of birds to novel environments. Proceedings of the National Academy of Sciences of the United States of America 102:5460-5465.

Sol, D., S. Bacher, S. M. Reader, and L. Lefebvre. 2008. Brain size predicts the success of mammal species introduced into novel environments. American Naturalist 172:S63-S71. 
Steiner, U. K. and J. Van Buskirk. 2008. Environmental stress and the costs of whole-organism phenotypic plasticity in tadpoles. Journal of Evolutionary Biology 21:97-103.

Steinger, T., B. A. Roy, and M. L. Stanton. 2003. Evolution in stressful environments II: Adaptive value and costs of plasticity in response to low light in Sinapis arvensis. Journal of Evolutionary Biology 16:313-323.

Sultan, S. 2015. Organism and Environment: Ecological Development, Niche Construction, and Adaptation. Oxford University Press, Oxford.

Sultan, S. E. 2021. Phenotypic plasticity as an intrinsic property of organisms. In D. W. Pfennig, ed., Phenotypic Plasticity and Evolution: Causes, Consequences, Controversies. CRC Press, Boca Raton, FL.

Sultan, S. E. and H. G. Spencer. 2002. Metapopulation structure favors plasticity over local adaptation. American Naturalist 160:271-283.

Szpiro, S. F. A. and M. Carrasco. 2015. Exogenous attention enables perceptual learning. Psychological Science 26:1854-1862.

Tebbich, S., B. Fessl, and D. Blomqvist. 2009. Exploration and ecology in Darwin's finches. Evolutionary Ecology 23:591-605.

Thuy, J. L., J. M. VandenBrooks, and M. J. Angilletta. 2016. Developmental plasticity evolved according to specialist: Generalist tradeoffs in experimental populations of Drosophila melanogaster. Biology Letters 12:20160379.

Travisano, M. and R. E. Lenski. 1996. Long-term experimental evolution in Escherichia coli 4. Targets of selection and the specificity of adaptation. Genetics 143:15-26.

Tufto, J. 2015. Genetic evolution, plasticity, and bet-hedging as adaptive responses to temporally autocorrelated fluctuating selection: A quantitative genetic model. Evolution 69:2034-2049.

Van Buskirk, J. and U. K. Steiner. 2009. The fitness costs of developmental canalization and plasticity. Journal of Evolutionary Biology 22:852-860.

Van Dyken, J. D. and M. J. Wade. 2010. The genetic signature of conditional expression. Genetics 184:557-570.

Van Kleunen, M. and M. Fischer. 2001. Adaptive evolution of plastic foraging responses in a clonal plant. Ecology 82:3309-3319.

Van Tienderen, P. H. 1991. Evolution of generalists and specialists in spatially heterogenous environments. Evolution 45:1317-1331.

Veening, J. W., W. K. Smits, and O. P. Kuipers. 2008. Bistability, epigenetics, and bet-hedging in bacteria. Annual Review of Microbiology 62:193-210.

Via, S. 1993. Adaptive phenotypic plasticity: Target or by-product of selection in a variable environment? American Naturalist 142:352-365.

Watson, R. A. and E. Szathmary. 2016. How can evolution learn? Trends in Ecology \& Evolution 31:147-157.

Weinig, C., J. Johnston, Z. M. German, and L. M. Demink. 2006. Local and global costs of adaptive plasticity to density in Arabidopsis thaliana. American Naturalist 167:826-836.

West-Eberhard, M. J. 2003. Developmental Plasticity and Evolution. Oxford University Press, New York.

Westneat, D. F., L. J. Potts, K. L. Sasser, and J. D. Shaffer. 2019. Causes and consequences of phenotypic plasticity in complex environments. Trends in Ecology \& Evolution 34:555-568.

Whitlock, M. C. 1996. The red queen beats the jack-of-all-trades: The limitations on the evolution of phenotypic plasticity and niche breadth. American Naturalist 148:S65-S77.

Workman, A. D., C. J. Charvet, B. Clancy, R. B. Darlington, and B. L. Finlay. 2013. Modeling transformations of neurodevelopmental sequences across mammalian species. Journal of Neuroscience 33:7368-7383. 